\title{
INTRA-CLASS CONSTRAINED SPARSE REPRESENTATION WITH GRADIENT FEATURE FOR OCCLUDED FACE RECOGNITION
}

\author{
Qinqin Ma and Xiang Ma \\ School of Information Engineering, Chang'an University, Xi'an 710048, China
}

\begin{abstract}
A novel occlusion face recognition method based on gradient feature and intra-class constrained sparse representation classification is proposed in this paper. We propose a second-order gradient feature to extract the texture and contour of images, which is more robust to occlusion than the original pixel of the face image. Intra-class constraint sparse representation is designed to make the test image close to the specific class by minimizing intra-class error. Furthermore, intra-class error is used as classification criteria. Experiments on the Extended Yale B and AR face databases prove the superiority and robustness of the proposed method for occluded face recognition, especially for the real world occlusion.
\end{abstract}

\section{KEYWORDS}

Occluded Face Recognition, Intra-Class Constraint

\section{INTRODUCTION}

Face recognition technology has achieved remarkable achievements and has been applied in many aspects of social life, including online verification, secure payment, access control system and so on. In the real situation, face occlusion is very common and the factors that cause occlusion are various, for instance, external obstacles such as scarves and glasses, as well as shadow occlusion caused by illumination. Figure 1 shows face images with different types of occlusion. Diversified occlusion types, uncertain occlusion areas, and random occlusion positions have a huge impact on the accuracy of face recognition. Considering the difficulty of face recognition due to the randomness and complexity of occlusion, this paper focuses on the problem of occlusion face recognition.

In recent years, occlusion face recognition methods can be roughly divided into two categories: robust feature extraction and robust classifier. The core idea of robust feature extraction methods is to separate the occlusion region from the overall face images and reduce or even ignore the weight of the occlusion region in the recognition process. A key-points descriptor of the Gabor ternary pattern for partial face recognition was proposed (Liao et al., 2013). Lu et al. (2015) reduce the difference in the feature level between face images by learning a compact binary face descriptor. An directional illumination estimation set algorithm (Cheng et al., 2017) was proposed to improve the method of multiscale principal contour direction (Cheng et al., 2010), which greatly alleviates the false contours of shadow edges and preserves the basic characteristics of the face. Robust feature extraction methods achieve good results for the certain type of occlusion, but can not fundamentally remove occlusion from the face image. In the existing feature extraction methods, region division and weight setting are still issues that need to be explored.

The second category mainly focuses on training robust classifiers. Wright et al. (2009) have proposed a sparse representation classification (SRC) method to represent test samples through sparse linear combination of training dictionaries. The cooperative representation classification (CRC) (Zhang et al., 2011) and the probability CRC (ProCRC) (Cai et al., 2016) are more conducive than the SRC. The robust version of ProCRC (R-ProCRC) is valid for occlusion. Since the occlusion portion contains structural information, the SRC does not effectively simulate the occlusion portion of face images. A structural constraint based on the dictionary learning method is proposed to deal with occlusion (Ou et al., 2014). Recently, Huang et al. (2017) proposed a kernel extended occlusion dictionary to improve efficiency. In real-world occlusion situations, 
since occlusion pixels have spatial continuity rather than being isolated from each other, Zhou et al. (2009) used Markov random field to describe the spatial correlation of occlusion pixels. The half-quadratic based iterative minimization method used a semi-quadratic minimization framework and iteratively restored occluded data (He et al., 2014). Inspired by robust principal component analysis, extracting sparse error of robust PCA is used to compute descriptors for face recognition (Luan et al, 2014). Li (2013) proposed a structured sparse error coding algorithm. A further improvement is to establish a model based on structured sparse error coding with discriminant error, structural error and occlusion support (Liang et al., 2015). At present, the use of sparse representation methods to deal with the face occlusion problem has received great attention, but further improvement of the recognition performance is needed.
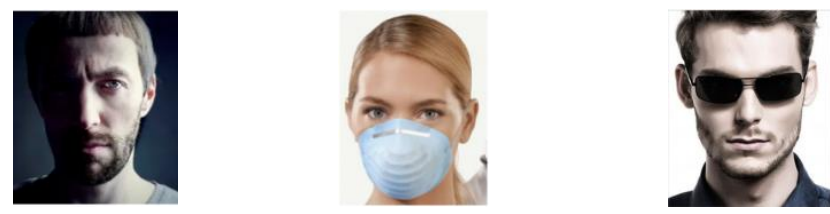

Figure 1. Face images with different occlusion types, from left to right: shadow occlusion, mask occlusion, sunglasses occlusion

Inspired by the above two types of methods, we propose a gradient feature-based intra-class constraint sparse representation method that combines robust features with robust classification. We extract the second-order gradient feature of the face image as the basis of recognition process. The gradient feature can better describe the edge and texture, and has better occlusion robustness than the original pixels of the face image. The intra-class error is finally used as a classification criterion. The contributions of this paper are as follows:

1) Fully exploit the performance of robust feature extraction and the error representation and detection capabilities of robust classifiers by combining robust feature extraction techniques with robust classifiers.

2) The second-order gradient feature is used to describe the edge and texture of the face image, which has better occlusion robustness than the original pixels of the face image, and contributes to the understanding and analysis of the face image.

3) Intra-class constraint is introduced in the sparse representation classification method to expand inter-class differences and reduce intra-class variations.

\section{PROPOSED METHOD}

In this part, we propose an intra-class constrained sparse representation method based on gradient feature, which specially describes the process of extracting second-order gradient feature and how to model the intra-class constrained sparse representation. The framework of the proposed algorithm is shown in Figure 2.

\subsection{Second-Order Gradient Feature}

Since the gradient feature can better describe the edge and texture of the face image, we propose to replace the original pixel of face images with the gradient feature. The second-order vector gradient $\nabla^{2} I$ of the face image is calculated by the following formula:

$$
\nabla^{2} I=\left[G_{r 2}, G_{c 2}\right]^{T}=\left[\frac{\partial^{2} I}{\partial r^{2}}, \frac{\partial^{2} I}{\partial c^{2}}\right]^{T}
$$

where $r$ and $c$ represent the row and column direction of the face image $I . G_{r 2}$ and $G_{c 2}$ respectively represent the second-order gradient of the horizontal and vertical direction.

$$
g(\theta(I))=1+\frac{2}{1+e^{s^{*}(\theta(I)-v)}}
$$


where $s$ and $v$ represent the scaling and offset factor, respectively. In order to ensure the convergence of the optimization process, the formula (2) is used to map the second-order gradient direction of the face image into second-order gradient features $g(\theta(I))$. When the gradient direction $\theta(I)=G_{r 2} / G_{c 2}$ approaches infinity, the mapping function can make the second-order gradient feature close to a constant value.

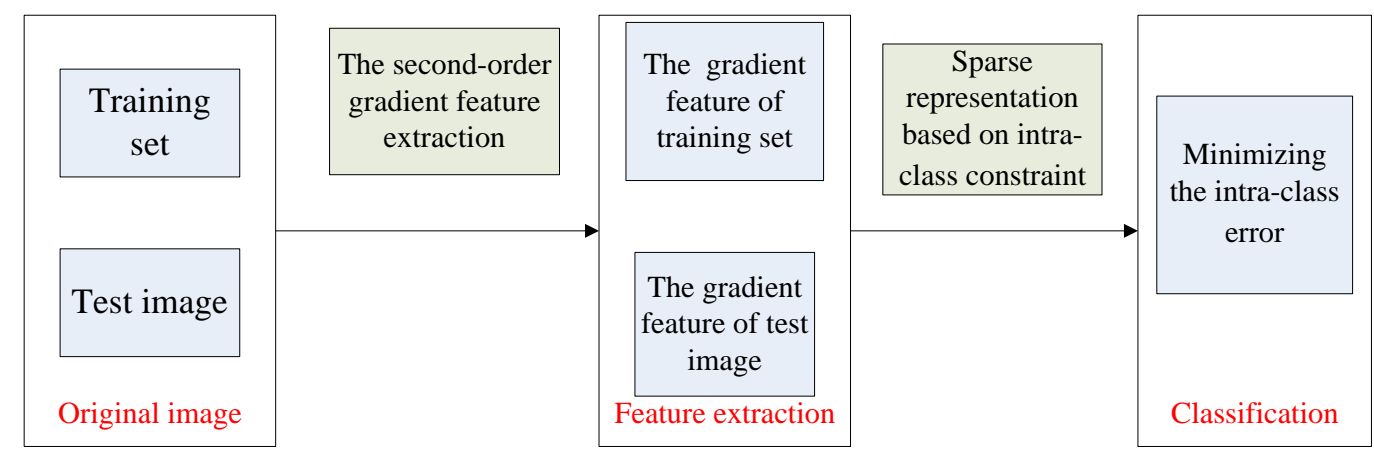

Figure 2. The framework of the proposed algorithm

The second-order gradient feature can describe the edge and texture information of the face image, and has better occlusion robustness than the image intensity domain feature. By extracting the contour and texture, structural features of the face image can be obtained, which provide a robust feature for subsequent classification and reduce the influence of occlusion on face recognition. Therefore, we propose to use the second-order gradient feature information instead of the intensity domain feature of the original image as the basis for the sparse representation classification.

\subsection{Intra-Class Constrained Sparse Representation}

SRC is a popular classification method and many extensions have been proposed. It mainly represents the test image by sparse linear combination of training samples, and uses the reconstruction error to achieve classification. It is assumed that the training set includes face images belonging to $\mathrm{K}$ classes, denoted as $X=\left[X_{1}, \ldots, X_{k}, \ldots ., X_{K}\right]$, where $X_{k}$ represents the k-th class face sample matrix of size $d \times m, m$ represents the number of k-th class samples and $d$ represents the dimension of the face image column vector. The test image $y$ can be represented as $y=X w$ by the SRC method, where $w$ is a sparse representation vector. Since the $l_{0}$-norm cannot be directly solved, the sparse representation vector is calculated by relaxing it as a $l_{1}$-norm convex optimization problem:

$$
\min _{w} \lambda\|w\|_{1} \text { s.t. } y=X w
$$

where $\lambda$ is a constant. According to the proposed second-order gradient feature extraction method in Section 2.1, the gradient feature of training set and test images can be obtained as $G=\left[G_{1}, \ldots, G_{k}, \ldots, G_{K}\right]$ and $g(\theta(y))$, respectively. For convenience, we define $g(\theta(y))$ as $y_{g} . G_{k}$ indicates the gradient feature of the k-th class sample $X_{k}$. We replace the original image pixels with the second-order gradient feature obtained in Section 2.1, and the formula based on the sparse representation of gradient feature is:

$$
\min _{w} \lambda\|w\|_{1} \text { s.t. } y_{g}=G w
$$

The $l_{1}$-norm in the SRC method guarantees the sparsity of the linear representation vector. In order to make the reconstruction error of the test image as small as possible, we improve the performance of face recognition by introducing an intra-class constraint. The equation for the sparse representation method based on intra-class constraint with gradient feature is defined by:

$$
\min _{w} \lambda\|w\|_{1}+\frac{\beta}{K} \sum_{k=1}^{K}\left\|G w-G_{k} w_{k}\right\|_{2}^{2} \quad \text { s.t. } y_{g}=G w
$$


where $w=\left[w_{1} ; w_{2} ; \ldots ; w_{k} ; \ldots ; w_{K}\right]$ and $w_{k}$ is a representation coefficient corresponding to $G_{k} . \lambda$ and $\beta$ are balance factors for adjusting sparsity and intra-class constraint. Intra-class constraint can make test images close to the specific class by minimizing intra-class errors while maintaining sparsity. The intra-class constraint continuously adjusts the representation coefficient $w_{k}$ according to the $\mathrm{k}$-th class gradient feature to obtain the optimal solution.

We use the augmented Lagrangian multiplier method to optimize equation (5) to obtain the solution of $w$ :

$$
(\hat{w})=\arg \min _{w}\left\{\left\|y_{g}-G w\right\|_{2}^{2}+\lambda\|w\|_{1}+\frac{\beta}{K} \sum_{k=1}^{K}\left\|G w-G_{k} w_{k}\right\|_{2}^{2}\right\}
$$

After obtaining the solution of the sparse representation vector $w$, the intra-class error $r(y)$ is finally used as a classification criterion:

$$
r(y)=\arg \min _{k}\left\{\left\|G w-G_{k} w_{k}\right\|_{2}^{2}\right\}
$$

The proposed method combines the advantages of robust feature and robust classifier. The second-order gradient feature extracts the edges and contours of face images, which helps to reduce the impact of occlusion on recognition performance. The SRC method based on intra-class constraint simultaneously guarantees sparsity and intra-class constraint, making the test image close to the specific class and minimizing the reconstruction error. In the next section, we will verify the effectiveness and robustness of the proposed method through sufficient experiments.

\section{EXPERIMENTAL RESULTS}

In this section, we evaluated the effectiveness and robustness of the proposed method for occlusion face recognition by conducting experiments on Extended Yale B (Georghiades et al, 2001) and AR face database (Martinez and Benavente, 1998). To illustrate the superiority of the proposed method, the proposed method was compared with some related representative methods, including SRC, CRC, ProCRC, the robust version of SRC(R-SRC) (Yang and Zhang, 2011) and R-ProCRC. For fair comparison, all methods of comparison use the optimal parameter settings. The two balance factors $\lambda$ and $\beta$ in the proposed method are determined by cross-validation, so we set $\lambda=1$ and $\beta=0.01$.

\subsection{Experiments on Extended Yale B Face Database}

In order to verify the recognition effect of the proposed algorithm on the occlusion factor, the algorithm was tested on the Extended Yale B face database of Yale University. There are 38 different categories of face images in the Extended Yale B face database, each class containing 64 different lighting conditions. All face images used in the experiment are manually aligned and cropped, leaving only the face area. And the resolution of face images is also adjusted to $32 \times 32$. In this experiment, each class randomly selects 30 images as the training set, and the remaining images as the test set. In addition, the recognition rate is the average of 5 test results, and each time different training set and test set were randomly selected. We determine the second-order gradient feature parameters $s=2.8$ and $v=0.28$ through experiments. Each image in the test set is randomly occluded by uncorrelated images, as shown in Figure 3. The occlusion ratio from left to right is $10 \%, 20 \%, 30 \%, 40 \%$, respectively.
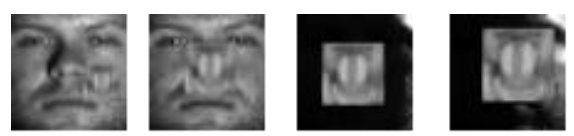

Figure 3. Sample images with different sizes of occlusion. From left to right, the ratio of occlusion is $10 \%, 20 \%, 30 \%$, $40 \%$, respectively 
Table 1 shows the recognition rates of different methods under different occlusion rates. It can be seen from Table 1 that compared with other methods, the proposed method achieves the best recognition results under different block sizes, which fully demonstrates the effectiveness of the proposed method. The performance of ProCRC decreases sharply with the increasing occlusion area, indicating that the ProCRC is sensitive to corruption and occlusion. R-ProCRC is an improved version of ProCRC, which achieves robustness to occlusion, and its recognition rate is only lower than the proposed method. However, the decline trend of R-ProCRC recognition performance is still relatively obvious compared to the proposed method as the occlusion ratio increases. The proposed method still achieves a recognition rate of $89.64 \%$ at the occlusion rate of $40 \%$, which proves that the occlusion factor has little effect on the proposed method. When the occlusion rate is 0 , the recognition rate of the proposed method is still the highest, indicating that the recognition performance of the proposed method is excellent regardless of whether the face image is occluded or not.

Table 1. Experimental results with different sizes of block occlusion on the Extended Yale B database

\begin{tabular}{llllll}
\hline Occlusion rate & 0 & $10 \%$ & $20 \%$ & $30 \%$ & $40 \%$ \\
\hline SRC & $95.97 \%$ & $89.84 \%$ & $83.06 \%$ & $73.61 \%$ & $62.33 \%$ \\
CRC & $96.83 \%$ & $93.65 \%$ & $85.13 \%$ & $72.80 \%$ & $60.02 \%$ \\
R-SRC & $96.28 \%$ & $90.36 \%$ & $86.13 \%$ & $78.44 \%$ & $69.79 \%$ \\
ProCRC & $97.65 \%$ & $81.93 \%$ & $72.77 \%$ & $63.70 \%$ & $54.65 \%$ \\
R-ProCRC & $98.22 \%$ & $97.76 \%$ & $90.87 \%$ & $83.42 \%$ & $75.47 \%$ \\
Proposed method & $99.69 \%$ & $98.51 \%$ & $97.88 \%$ & $96.38 \%$ & $89.64 \%$ \\
\hline
\end{tabular}

\subsection{Experiments on AR Face Database}

In the previous section, the robustness of the proposed method is tested mainly by adding occlusion to the face image. To further verify the applicability of the proposed method, we test it on the AR face database with real guise. There are 100 different classes in the AR database, including 50 males and 50 females. Each category has two subsets, and the two subsets are taken at different times. Each subset consists of 7 unoccluded face images, 3 sunglasses occluded images, and 3 scarves occluded images. All face images are preprocessed and their resolution is set to $42 \times 30$. In this experiment, 7 unoccluded face images of the first subset are used as the training set, while 6 sunglasses occlusion images and 6 scarf occlusion images from the two subsets are used as test sets, respectively. As shown in Figure 4, the training set samples, the glasses occlusion test samples, and the scarf occlusion test samples are sequentially arranged from top to bottom. For the AR face database, we adjust the parameters $s=7.3$ and $v=0.51$.

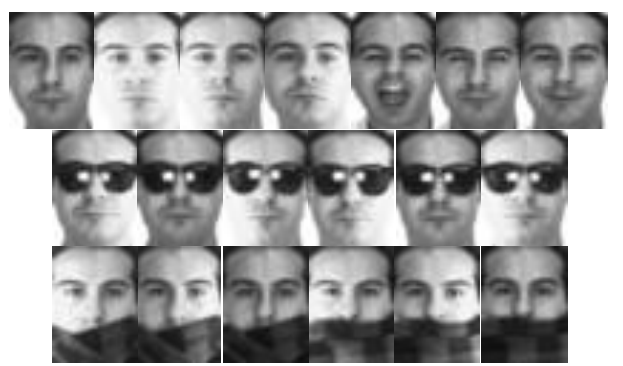

Figure 4. Sample diagram of AR face database (From top to bottom, the face images are from the training set, the glasses occlusion test set, and the scarf occlusion test set, respectively)

Table 2. Recognition rates of different methods on the AR face database

\begin{tabular}{lccc}
\hline Occlusion type & Glasses & Scarf & Average \\
\hline SRC & $67.50 \%$ & $61.83 \%$ & $64.67 \%$ \\
CRC & $68.83 \%$ & $63.00 \%$ & $65.92 \%$ \\
R-SRC & $70.33 \%$ & $64.33 \%$ & $67.33 \%$ \\
ProCRC & $53.33 \%$ & $49.83 \%$ & $51.58 \%$ \\
R-ProCRC & $71.67 \%$ & $65.00 \%$ & $68.34 \%$ \\
Proposed method & $87.00 \%$ & $78.00 \%$ & $82.50 \%$ \\
\hline
\end{tabular}


Table 2 shows the recognition rates of all methods for different occlusion types. The results in Table 2 show that all methods have better peformance on the glasses occlusion than the scarf occlusion. Because the scarf has the larger occlusion area, and it blocks some discriminative facial features. ProCRC has obtained the lowest recognition rate in all methods of comparison, indicating that it is sensitive to occlusion. The performance of CRC is slightly superior to SRC. The proposed method has obvious advantages, and it improves the recognition rate by about $14 \%$ compared with R-ProCRC. The experiments on the AR database fully demonstrate that the proposed method is also robust to real-world occlusion.

\section{CONCLUSION}

We propose a novel method using gradient feature and intra-class constrained sparse representation classification to solve the occlusion problem of face recognition. The second-order gradient feature is proposed as a robust feature for extracting the texture and contour of the face image. It is more robust than the original pixels of the face image and can effectively reduce the influence of occlusion. Intra-class constrained sparse representation is used as a robust classifier to minimize intra-class errors, making test images close to the specific class. Intra-class constraint achieves the purpose of expanding the differences between classes and narrowing the distance within the class. We use the intra-class error as a classification criterion. We have carried out sufficient experiments on the Extended Yale B face database and AR database, and compared the proposed method with some representative methods. Experimental results demonstrate the superiority and robustness of the proposed method for occlusion face recognition.

The proposed algorithm in this paper has achieved good recognition results on the standard face database. How to apply the proposed algorithm to face recognition in unconstrained natural scene is the research direction of future work.

\section{ACKNOWLEDGEMENT}

This work was supported in part by the National Natural Science Foundation of China under Grant 61771075, and in part by the China Fundamental Research Funds for the Central Universities under Grant 300102249203 .

\section{REFERENCES}

Cai S. et al, 2016. A probabilistic collaborative representation based approach for pattern classification. Proceedings of the IEEE Computer Society Conference on Computer Vision and Pattern Recognition. Las Vegas, USA, pp. 2950-2959.

Cheng Y. et al, 2010. Multiscale principal contour direction for varying lighting face recognition. In Electronics Letters. Vol. 46, No. 10, pp. 680-682.

Cheng Y. et al, 2017. Directional Illumination Estimation Sets and Multilevel Matching Metric for Illumination-Robust Face Recognition. In IEEE Access. Vol. 5, pp. 25835-25845.

Georghiades A.S. et al, 2001. From few to many: illumination cone models for face recognition under variable lighting and pose. In IEEE Transactions on Pattern Analysis and Machine Intelligence. Vol. 23, No. 6, pp. 643-660.

He R. et al, 2014. Half-quadratic based iterative minimization for robust sparse representation, In IEEE Transactions on Pattern Analysis and Machine Intelligence. Vol. 36, Vo. 2, pp. 261-275.

Huang K. et al, 2017. Learning Kernel Extended Dictionary for Face Recognition, In IEEE Transactions on Neural Networks and Learning Systems, Vol. 28, No. 5, pp. 1082-1094.

Li X. et al, 2013. Structured Sparse Error Coding for Face Recognition with Occlusion. In IEEE Transactions on Image Processing. Vol. 22, No. 5, pp. 1889-1900.

Liang R. et al, 2015. Mixed error coding for face recognition with mixed occlusions. Proceedings of the International Joint Conf. Artificial Intelligence, pp. 3657-3663.

Liao S. et al, 2013. Partial face recognition: alignment-free approach, In IEEE Transactions on Pattern Analysis and Machine Intelligence. Vol. 35, No. 5, pp. 1193-1205. 
International Conferences Interfaces and Human Computer Interaction 2019; Game and Entertainment Technologies 2019; and Computer Graphics, Visualization, Computer Vision and Image Processing 2019

Lu J. et al, 2015. Learning Compact Binary Face Descriptor for Face Recognition. In IEEE Transactions on Pattern Analysis and Machine Intelligence. Vol. 37, No. 10, pp. 2041-2056.

Luan X. et al, 2014. Extracting sparse error of robust PCA for face recognition in the presence of varying illumination and occlusion, Pattern Recognit. Vol. 47, No. 2, pp. 495-508.

Martinez A. and Benavente R., 1998. The AR face database. CVC Technical Report, No. 24.

$\mathrm{Ou}$ W. et al, 2014. Robust face recognition via occlusion dictionary learning. Pattern Recognition. Vol. 47, No. 4, pp. 1559-1572.

Wright J. et al, 2009. Robust face recognition via sparse representation, In IEEE Trans. Pattern Anal. Mach. Intell. Vol. 31, No. 2, pp. 210-227.

Yang J. and Zhang Y., 2011. Alternating direction algorithms for $\ell 1$-problems in compressive sensing. In SIAM journal on scientific computing. Vol. 33, No. 1, pp. 250-278.

Zhang L. et al, 2011. Sparse representation or collaborative representation: which helps face recognition? In: Proceedings of the IEEE International Conference Computer Vision. Barcelona, pp. 471-478.

Zhou Z. et al, 2009. Face recognition with contiguous occlusion using markov random fields. Proceedings of International Conference on Computer Vision. Kyoto, pp. 1050-1057. 